\title{
INFLUÊNCIA DO TIPO DE RAMO SOBRE O CRESCIMENTO E PRODUÇÃO DO CAJUEIRO-ANÃO-PRECOCE DE COPA SUBSTITUÍDA ${ }^{1}$
}

\author{
ADROALDO GUIMARÃES ROSSETTI² \& ANTONIO RENES LINS DE AQUINO²
}

\begin{abstract}
RESUMO - Visando a testar a influência do ramo fornecedor de propágulo para enxertia por borbulhia, no crescimento e produção do cajueiro-anãoprecoce, realizou-se um experimento na fazenda Itaueira, município de Canto do Buriti, Piauí. O experimento foi instalado em blocos ao acaso, com oito tratamentos: ramo em início de floração sem folha; ramo em início de floração com folha; ramo vegetativo sem folha; ramo vegetativo com folha; ramo com panícula seca sem folha; ramo com panícula seca com folha; ramo com flores abertas sem folha; ramo com flores abertas com folha; quatro repetições e de cinco a oito plantas por parcela. Utilizou-se um pomar de plantas de pé-franco, as quais foram decepadas e suas brotações enxertadas a pleno sol com propágulos do clone CCP 76. Avaliaram-se, na fase juvenil, o pegamento de enxertia, a altura da planta, a envergadura leste-oeste, e, na fase adulta, a produção: peso e número de castanha. A análise dos dados permitiu concluir que os melhores tratamentos, da fase juvenil, onde se verificou maior porcentagem de pegamento de enxertia (96\%), e as plantas que tiveram melhor desenvolvimento, foram os enxertados com propágulos de ramos com floração e flores abertas, independentemente de ter ou não folhas, seguidos dos em início de floração, nas mesmas condições. Na fase produtiva, os propágulos de ramos com floração, cujas produções não diferiram entre si, apresentaram-se mais produtivas e significativamente diferentes dos de ramos vegetativos e com panícula seca.
\end{abstract}

Termos para indexação: Enxertia por borbulhia, fontes de propágulos, substituição de copa, manejo cultural, Anacardium occidentale, L.

\section{INFLUENCE OF SHOOT TYPE ON THE GROWTH AND PRODUCTION OF DWARF CASHEW FROM TOP WORKING}

\begin{abstract}
A trial was conducted aiming to determine the influence of CCP 76 dwarf cashew shoot type as source of bud for grafting, in Canto do Buriti county, State of Piaui (Brazil). The experimental design was a randomized block with 8 treatments, 4 replicates and 5 to 8 plants per plot. The treatments were as follows: shoot without leaves and beginning flowering; shoot with leaves and beginning flowering; vegetative shoot without leaves; vegetative shoot with leaves; shoot with dried panicles and without leaves; shoot with dried panicles and with leaves; shoot with opened flowers and without leaves, and shoot with opened flowers and with leaves. The work was carried out in a plantation originally seed planted. After plants decapitation and flushing of new shoots top working was conducted under direct sun light. During the juvenile phase were evaluated the percentage of viable grafting, plant height and canopy length (east to west); for the adult phase was evaluated the production (weight and number of nuts). The results analysis revealed that in a juvenile phase the percentage of viable grafting of $96 \%$ and the best development was obtained from shoots with opened flowers, irrespective of having leaves or not, followed by shoots in the beginning of flowering, with or without leaves. For the adult phase the best results were obtained with buds from flowering shoots, without significant differences in production, but significantly different from vegetative shoots with dried panicles.
\end{abstract}

Index terms: budding, source of propagule, top working, crop management, Anacardium occidentale, L..

\section{INTRODUÇÃO}

A área plantada com o cajueiro-anão-precoce tem crescido acentuadamente. O porte baixo, que facilita a colheita, o manejo e a condução dos pomares, a precocidade, a maior produtividade em relação ao tipo comum e o crescente interesse ao aproveitamento do pedúnculo, tanto para consumo "in natura", como para a agroindústria, são os fatores que têm contribuído para o aumento da área plantada. Devido à expansão ter ocorrido com muita rapidez, houve inicialmente pouca disponibilidade de muda enxertada com material genético de boa qualidade. Esta pouca disponibilidade de mudas elevou o custo, o que ocasionou a formação de pomares de cajueiro anão precoce com mudas propagadas por semente, os quais se mostram com grande desuniformidade e baixa produtividade. Entre as graves conseqüências dessa desuniformidade, Crisóstomo et al. (1992) destacaram a heterogeneidade das plantas, das castanhas, das amêndoas e dos pedúnculos, com efeitos sobre o rendimento e produtividade da cultura. Barros et al. (1984) e Araújo \& Rodrigues (1988) estimaram que, de 30\% a 50\% desses cajueiros, são improdutivos ou apresentam baixa produtividade. Na busca de recuperar a rentabilidade desses pomares, a Embrapa Agroindústria Tropical, viabilizou a tecnologia da substituição de copa, que se tem revelado, conforme Rossetti et al. (1998), como excelente alternativa na recuperação desses pomares improdutivos. Trata-se de uma prática simples, barata e que pode ser realizada em qualquer época do ano, desde que haja propágulos adequados para enxertia. Este fato propicia grande flexibilidade de ajustes nas atividades da empresa agrícola, sem grandes prejuízos. Com a viabilização dessa tecnologia, por meio da enxertia por borbulhia em placa, a pleno sol, nas brotações crescidas das plantas decepadas, algumas pesquisas necessitarão ser realizadas. Estudar os melhores períodos para fazer-se a substituição de copa e escolher os ramos mais indicados para fornecerem as borbulhas para enxertia, são algumas investigações passíveis de buscar-se respostas.

O presente trabalho teve por objetivo identificar os melhores ramos fornecedores de borbulhas, avaliar o pegamento de enxertia e testar a sua eventual influência sobre o crescimento e produção do cajueiro-anão-precoce, na substituição de copa.

\section{MATERIALEMÉTODOS}

O trabalho foi conduzido na fazenda Itaueira, pertencente à CEMAG - Ceará Máquinas Agrícolas S.A., localizada no município de Canto do Buriti, no Estado do Piauí, situada à latitude de $8^{\circ} 17^{\prime}$ e longitude de $43^{\circ} 21^{\prime}$, de agosto de 1990 a dezembro de 1995 , num plantio com dois anos de idade. A área utilizada apresenta, segundo Ramos et al. (1997), as seguintes características: Latossolo amarelo, de textura média e baixa fertilidade natural, bem drenada, topografia plana, altitude de 500 m, clima BSkw, semi-árido quente, com chuvas de verão distribuídas entre setembro e abril, e precipitação média anual de $790 \mathrm{~mm}$, temperatura média de $26,4^{\circ} \mathrm{C}$ e $60 \%$ de umidade relativa média. O experimento foi instalado em área já implantada com material de pé-franco, originado de semente de cajueiro-anão-precoce, cuja área e plantas foram escolhidas conforme metodologia proposta por Rossetti \& Barros (1996). As plantas selecionadas foram decepadas conforme Rossetti et al. (1993). Sessenta dias após o decepamento das plantas, realizou-se um desbaste nas brotações e foram selecionadas, em cada planta, as duas mais vigorosas. As brotações foram distribuídas, tanto quanto possível, simetri-

1 (Trabalho 061/2002). Recebido: 08/03/2002. Aceito para publicação: 26/09/2002.

2 Pesquisadores Embrapa Agroindústria Tropical (CNPAT), Rua Dra. Sara Mesquita, 2270 - Planalto Pici, Caixa Postal 3761, CEP 60511-110 Fortaleza-CE.

Telefone (0xx85) 299-1835 adroaldo@cnpat.embrapa.br e renes@cnpat.embrapa.br 
camente, em relação ao tronco da planta, conforme sugerem Rossetti et al. (1998). Nessas brotações, foram enxertadas, a pleno sol, borbulhas do clone CCP76. As borbulhas foram obtidas de oito tipos de ramos: (1) ramo em início de floração e sem folha; (2) ramo em inicio de floração e com folha; (3) ramo vegetativo e sem folha; (4) ramo vegetativo e com folha; (5) ramo com panícula seca e sem folha; (6) ramo com panícula seca e com folha; (7) ramo com flores abertas e sem folha; (8) ramo com flores abertas e com folha. O experimento foi instalado em delineamento de blocos casualizados, com quatro repetições. Em cada parcela, foram escolhidas de cinco a oito plantas úteis. Nas brotações selecionadas, a enxertia por borbulhia em placa foi realizada em novembro de 1990, por dois enxertadores experientes.

O manejo da área experimental foi o mesmo adotado pela $\mathrm{Fa}$ zenda, para todo o pomar.

Os efeitos dos tratamentos foram avaliados a partir da fase juvenil, por meio de variáveis de vigor, em que foram medidas a porcentagem de pegamento de enxertos, a altura de planta e a envergadura leste-oeste, conforme Crisóstomo et al. (1992). Na fase produtiva, a partir do segundo ano de produção, durante quatro anos, avaliaram-se o peso e o número de castanhas. Na Tabela 1, são apresentados os resultados da análise de variância dos componentes, para todas as variáveis avaliadas, e a comparação dos tratamentos, apresentada na Tabela 2, foi feita pelo teste de Ryan-Einot-Gabriel-Welsch (regwq), equivalente ao teste de Tukey para dados não-balanceados, a $\mathrm{p}<0,05$ de probabilidade, a fim de que se comparassem todos os contrastes de duas médias. A comparação de dois grupos de tratamentos foi feita pelo teste de Duncan, a $\mathrm{p}<0,05$ de probabilidade.

\section{RESULTADOSE DISCUSSÃO}

Dez dias após o decepamento, todas as plantas já emitiam brotações intensamente. Essas brotações eram, em grande maioria, em torno de todo o caule. No grupo de tratamentos em que os propágulos foram fornecidos por ramos em floração, a porcentagem de pegamento da enxertia ocorreu em cerca de $96,00 \%$ das plantas, com diferença significativa a p $<0,05$ de probabilidade, pelo teste de Duncan, em relação ao grupo dos demais, onde o pegamento médio foi de $85,25 \%$. Considerouse, para esse efeito, a planta com, pelo menos, um enxerto pego, embora, em grande parte delas, tenha ocorrido pegamento de todos os enxertos feitos. Esse percentual de pegamento, superior aos cerca de $86 \%$ obtidos no preparo de mudas enxertadas em viveiro, pelo mesmo método de borbulhia em placa, deveu-se, provavelmente, ao vigor e à juvenilidade das plantas em que, já com o sistema radicular no local definitivo, passa água e nutrientes minerais em ótimas quantidades à nova parte aérea. Pelas estimativas de variâncias apresentadas na Tabela 1, verifica-se que as maiores variações ocorreram para porcentagem de pegamento de enxertos, cuja maior contribuição para isso foi dada pelos tratamentos enxertados com borbulhas de ramos vegetativos, e para número de castanhas, o que é normal para o cajueiro. As plantas com nova copa apresentaram excelente uniformidade, apesar de haver clara distinção entre dois grupos: um das enxertadas com gemas de ramos com floração e outro com gemas de ramos vegetativos e de panícula seca, como se observa na Tabela 2. Os tratamentos, cujos propágulos foram fornecidos por ramos vegetativos, foram os que tiveram pegamento mais baixo, mas sem diferença significativa entre si. Nas brotações enxertadas com propágulos provenientes de ramo com panícula seca, apesar de a porcentagem de pegamento ter sido ligeiramente superior aos provenientes de ramos vegetativos, a diferença não foi estatisticamente significativa. Quando, porém, comparados com os de ramos em floração, observou-se diferença significativa, a $\mathrm{p}<0,05$ de probabilidade, como se vê na Tabela 2. Esses resultados estão de acordo com os obtidos por Embrapa (1991), em que os melhores índices de enxertia de mudas de cajueiro foram conseguidos com borbulhas provenientes de ramos com panícula desenvolvida e os piores, com as de ramos com panícula seca.

Os resultados obtidos para a variável produção, medida pelo peso e número de castanhas, foram bastante similares entre si, como se vê Tabela 2. Isso ocorreu, devido à correlação positiva que há entre essas variáveis, principalmente entre envergadura leste-oeste e produção, conforme enfatizam Azevedo et al. (1998). Verifica-se, ainda, que as melhores produções ocorreram no grupo de plantas enxertadas com borbulhas provenientes de ramos com floração e as menores nas enxertadas com borbulhas provenientes de ramos vegetativos e com panícula seca. Neste grupo, a produção mais baixa foi verificada nas plantas enxertadas com propágulos fornecidos por ramos vegetativos, sem, contudo, haver diferenças significativas, quando comparadas às enxertadas com borbulhas de ramos com panícula seca, mesmo com produções ligeiramente superiores.

Esses resultados fortalecem o uso da substituição de copa em cajueiros. Cada vez mais, essa técnica torna-se eficaz e serve como alternativa viável para recuperar a uniformidade e produtividade dos pomares formados por plantas propagadas por semente.

TABELA 1 - Análise de variância das variáveis Altura de planta, Envergadura leste-oeste, em metros [Altura (m)], [Env.L-O(m)], Peso de castanha, em kg/ha [Pcas ( $\mathrm{kg} / \mathrm{ha})$ ] e Número de castanhas (Ncas) e porcentagem de pegamento de enxertos $[\operatorname{Pen} x(\%)]$.

\begin{tabular}{lrccccc}
\hline Fonte de varia 0 & GL & \multicolumn{5}{c}{ Quadrados Médios } \\
\cline { 2 - 7 } & & Altura $(\mathrm{m})$ & Env.L-O(m) & Pcas (kg/ha) & Ncas & Penx(\%) \\
\hline Bloco & 3 & - & - & - & - & - \\
Tratamento & 7 & $0,1405^{*}$ & $0,4497^{*}$ & $0,7937^{*}$ & $39.537,3029^{*}$ & $132,5671^{*}$ \\
Resíduo & 151 & 0,0584 & 0,1797 & 0,3113 & 6882,6180 & 43,1815 \\
Média & & 1,45 & 1,54 & 873,72 & 7280,00 & 96,01 \\
\hline
\end{tabular}

* Diferença estatisticamente significativa a $\mathrm{p}<0,03$ de probabilidade, pelo teste $\mathrm{F}$.

TABELA 2 - Porcentagem de pegamento de enxerto [(Pegam \%)], Altura da planta, em metros [Altura (m)], Envergadura lesteoeste (Env. L-O) e produção [Peso de castanha (PCAS)] e [Número de castanha (NCAS)], na substituição de copa com propágulos fornecidos por diversos tipos de ramo.

\begin{tabular}{lccccc}
\hline Tratamento $^{*}$ & Pegam (\%) & Altura (m) & Env.L-O (m) & PCAS (kg/ha) & NCAS \\
\hline RIFSF & $96 \mathrm{a}$ & $1,98 \mathrm{a}$ & $2,03 \mathrm{a}$ & $1.076,00 \mathrm{a}$ & $8966 \mathrm{a}$ \\
RIFCF & $95 \mathrm{a}$ & $1,95 \mathrm{a}$ & $2,05 \mathrm{a}$ & $998,90 \mathrm{a}$ & $8324 \mathrm{a}$ \\
RCFASF & $97 \mathrm{a}$ & $1,96 \mathrm{a}$ & $2,00 \mathrm{a}$ & $1.057,05 \mathrm{a}$ & $8808 \mathrm{a}$ \\
RCFACF & $96 \mathrm{a}$ & $1,95 \mathrm{a}$ & $2,00 \mathrm{a}$ & $999,00 \mathrm{a}$ & $8325 \mathrm{a}$ \\
RCPSSF & $88 \mathrm{~b}$ & $0,93 \mathrm{~b}$ & $1,05 \mathrm{~b}$ & $748,90 \mathrm{~b}$ & $6240 \mathrm{~b}$ \\
RCPSCF & $86 \mathrm{~b}$ & $0,92 \mathrm{~b}$ & $1,03 \mathrm{~b}$ & $750,00 \mathrm{~b}$ & $6250 \mathrm{~b}$ \\
RVSF & $84 \mathrm{~b}$ & $0,93 \mathrm{~b}$ & $1,02 \mathrm{~b}$ & $689,90 \mathrm{~b}$ & $5749 \mathrm{~b}$ \\
RVCF & $83 \mathrm{~b}$ & $0,91 \mathrm{~b}$ & $1,02 \mathrm{~b}$ & $670,00 \mathrm{~b}$ & $5583 \mathrm{~b}$ \\
\hline
\end{tabular}

Médias seguidas da mesma letra não diferem entre si, a $p<0,05$ de probabilidade, pelo teste Regwq; * RIFSF Ramo em início de floração e sem folha; RIFCF Ramo em início de floração e com folha; RCFASF Ramos com flores abertas e sem folha; RCFACF Ramo com flores abertas e com folha; RCPSSF Ramo com panícula seca e sem folha; RCPSCF Ramo com panícula seca e com folha; RVSF Ramo vegetativo e sem folha; RVCF Ramo vegetativo e com folha.

\section{CONCLUSÕES}

1. Na substituição de copa em cajueiro-anão-precoce, os propágulos mais apropriados para enxertia por borbulhia em placa, são os provenientes de ramos com flores abertas e no início da floração.

2. Os propágulos provenientes de ramos com flores abertas, e no início da floração, apresentaram maior porcentagem de pegamento de enxertos.

3. As plantas enxertadas com propágulos de ramos com flores abertas, e no início da floração, propiciaram maior produção nas plantas de copa substituída. 


\section{AGRADECIMENTO}

Os autores agradecem à Fazenda Itaueira, pelo apoio e colaboração para a realização deste trabalho.

\section{REFERÊNCIASBIBLIOGRÁFICAS}

ARAÚJO, J. P. P. de; RODRIGUES, S. C. Sistema de seleção de sementes de cajueiro para plantio: fator de produtividade. Fortaleza: EMBRAPA-CNPCa, 1988. 4p. (Caju Informativo, n.1).

AZEVEDO, D. M. P. de; CRISÓSTOMO, J. R.; ALMEIDA, F. C. G.; ROSSETTI, A. G. Estimates of genetic correlations and correlated responses to selection in cashew (Anacardium occidentale, L.). Genetics and Molecular Biology, Ribeirão Preto, v. 21, n. 3, p.399402, 1998.

BARROS, L. de M.; ARAÚJO, F. E. de; ALMEIDA, J. I. L. de; TEIXEIRA, L. M. S. A cultura do cajueiro-anão. Fortaleza: EPACE, 1984. 67p. (Documentos, 3).

CRISÓSTOMO, J. R.; ALMEIDA, J. I. L. de; GADELHA, J. W. R.; FILIPE, E. M. Orientação para o plantio de cajueiro em relação aos pontos cardeais. Fortaleza: EMBRAPA-CNPAT, 1992. 2p. (Comunicado Técnico, n.3).

CRISÓSTOMO, J. R.; GADELHA, J. W. R.; ARAÚJO, J. P. P. de; BAR-
ROS, L. de M. Conseqüências do plantio de sementes oriundas de plantas enxertadas ("clones") ou de plantas de "pé-franco" de cajueiro. Fortaleza: EMBRAPA-CNPAT, 1992.4p. (Caju Informativo, n.3).

EMBRAPA - Centro Nacional de Pesquisa de Caju. Relatório técnico anual do Centro Nacional de Pesquisa de Caju 1989-90. Fortaleza, 1991.84p.

RAMOS, A. D.; FROTA, P. C. E.; LIMA, A. A. C.; OLIVEIRA, F. N. S. Solos cultivados com cajueiro: características e limitações. Fortaleza: EMBRAPA-CNPAT, 1997. 48p. (Documentos, 21).

ROSSETTI, A. G.; BONASPETTI, E.; CORRÊA, M. P. F. Substituição de copa em cajueiros jovens como alternativa para pomares improdutivos. Fortaleza: EMBRAPA-CNPAT, 1993. 4p. (Caju Informativo, n.2).

ROSSETTI, A. G.; BARROS, L. de M. A methodology to make use of previously planted areas for field experiments with perennial trees. In: REUNIÃO ANUAL DA REGIÃO BRASILEIRA DA SOCIEDADE INTERNACIONAL DE BIOMETRIA, 41., 1996, São José do Rio PretoSP. Programa e Resumos: São José do Rio Preto: IB ILCE-UNESP, 1996. p.43.

ROSSETTI, A. G.; CORRÊA, M. P. F.; PINHEIRO, D. M. Recuperação de pomares jovens de cajueiro-anão-precoce propagados por semente. Revista Brasileira de Fruticultura, Cruz das Almas, v.20, n.2, p.202205, ago. 1998. 